\title{
Comparison of Simultaneous and Sequential Two-View Registration for 3D/2D Registration of Vascular Images
}

\author{
Chetna Pathak $^{1}$, Mark Van Horn ${ }^{1}$, Susan Weeks ${ }^{2}$, and Elizabeth Bullitt ${ }^{1}$ \\ ${ }^{1}$ Department of Surgery, University of North Carolina, Chapel Hill, NC \\ http: //casilab.med.unc.edu/ \\ ${ }^{2}$ Department of Radiology, University of North Carolina, Chapel Hill, NC \\ \{cpathak, mark_vanhorn, sue_weeks, bullitt\}@med.unc.edu
}

\begin{abstract}
Accurate 3D/2D vessel registration is complicated by issues of image quality, occlusion, and other problems. This study performs a quantitative comparison of $3 \mathrm{D} / 2 \mathrm{D}$ vessel registration in which vessels segmented from preoperative $\mathrm{CT}$ or MR are registered with biplane $\mathrm{x}$-ray angiograms by either a) simultaneous two-view registration with advance calculation of the relative pose of the two views, or b) sequential registration with each view. We conclude on the basis of phantom studies that, even in the absence of image errors, simultaneous two-view registration is more accurate than sequential registration. In more complex settings, including clinical conditions, the relative accuracy of simultaneous two-view registration is even greater.
\end{abstract}

\section{Introduction}

The objective of $3 \mathrm{D} / 2 \mathrm{D}$ registration is to align spatial data to projective data. Given a $3 \mathrm{D}$ model and its $2 \mathrm{D}$ projection, 3D/2D registration determines the pose (orientation and position) of the model at which its $2 \mathrm{D}$ image was taken. This paper discusses the registration of $3 \mathrm{D}$ vessels, segmented preoperatively from computed tomographic (CT) or magnetic resonance (MR) images, to biplane, $\mathrm{x}$-ray angiograms.

The driving clinical problem is the Transjugular Intrahepatic Portosystemic Shunt (TIPS) procedure, which creates a channel between the portal and hepatic veins [1]. We are currently developing an image-guided system for this procedure. One of the major challenges has been achieving accurate 3D/2D registration under conditions in which the x-ray angiograms are noisy and contain severe projection overlap (Fig. 1).

Several groups have described effective methods of 3D/2D vascular registration $[2,3,4,5]$. The purpose of the current paper is not to evaluate a specific registration metric, but rather to compare the efficacy of simultaneous, two-view registration with sequential registration. This issue has received little attention, although one paper notes in passing that simultaneous, two-view registration appears more effective than single-view registration [6]. However, no quantitative assessment or detailed analysis was provided.

This study uses phantom and clinical data to evaluate accuracy in registering a presegmented, 3D vessel model to biplane fluoroscopic images under two conditions. In the first, the relationship between cameras capturing the two views is determined in advance and the 3D model is registered simultaneously to the two views. In the second, the $3 \mathrm{D}$ model is registered sequentially and independently to each view. 

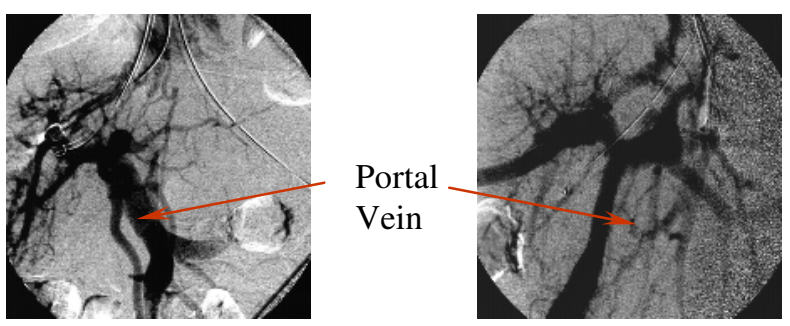

Fig. 1. Sample AP (left) and lateral (right) portograms used in the TIPS procedure. Note the thickness of the vessels and the projection overlap.

We conclude that the simultaneous approach is more accurate than the sequential approach even under ideal conditions. In the presence of image errors, the difference between the performance of the two methods increases. Although this report employs a particular registration metric [5], the findings in regard to sequential/simultaneous registration should be applicable to $3 \mathrm{D} / 2 \mathrm{D}$ registration methods using other metrics.

\section{Methods}

\subsection{Phantom Studies}

The purpose of the phantom studies was to evaluate registration accuracy under conditions of known ground truth and varying image quality. All tests were performed in blinded fashion, with the individual performing the registration unaware of the 3D pose of the vessels until after study completion.

Simulated angiograms were created by generating projections of four different portal venous trees, each segmented from the CT or MR of a different patient, and each containing 7-15 vessels. The fields of view ranged from $9^{\circ}$ to $16.5^{\circ}$, and the relative angle between the two views ranged from $80^{\circ}$ to $90^{\circ}$, with arbitrary placement and rotation of the 3D model within the imaged field. Two sets of pseudoangiogram pairs were generated for each of the four vascular models, using different model poses and different combinations of camera intrinsics. For each 3D vessel point, a circle was projected upon the view-plane with radius calculated using the vessel radius and rules of projection geometry. Using a combination of buffers and summing image intensities, the generated images simulate angiograms with vessel overlap (Fig. 2).

For simultaneous registration, the operator was given the camera intrinsics and the relationship between the two views, and registration was performed on both views simultaneously, giving one set of registration matrices for each view. For sequential registration, the operator was given each view's camera intrinsics and registration was performed individually, also giving one set of registration matrices for each image. Single-view registrations are independent and the order in which they are carried out unimportant. Both AP and lateral views were registered in order to make a complete comparison with results of simultaneous registration. The initial estimate of 3D model pose was up to $16^{\circ}$ off and up to $65 \mathrm{~mm}$ away from the actual position of the model. 
3D/2D registration was performed using a metric that optimizes a view-plane based disparity measure based on the iterative closest point algorithm between the $3 \mathrm{D}$ vessel skeletons and the skeletons of the vessel projections seen on angiograms [5]. Three different phantom studies were performed:

1) Ideal case pseudoangiograms: Registrations of the 8 image pairs with their respective $3 \mathrm{D}$ models were evaluated under conditions of projection overlap, but without added noise and with perfect one-to-one correspondence between vessels of the 3D model and their projections on pseudoangiograms. Fig. 2 illustrates a perspective projection of the 3D model and its AP and lateral pseudoangiograms.

2) Noisy pseudoangiograms: This study was identical to the one above, but with Gaussian noise of standard deviation 2.5 added to the pseudoangiograms. The addition of noise both obscures smaller vessels and can confuse the determination of vessel skeletons (Fig. 3).

3) Noisy pseudoangiograms without one-to-one vessel correspondence: This study was identical to the two above, but with the deletion of 3-8 branches from the 3D model. This situation provides a partial simulation of the actual clinical condition, in which a noisy x-ray angiogram can show vessels that the $3 \mathrm{D}$ model does not. Similarly, the 3D model may contain vessels that are not visible in the angiogram.

Registration accuracy was measured by comparing the location of each point in the 3D model following registration with its known location during synthetic image
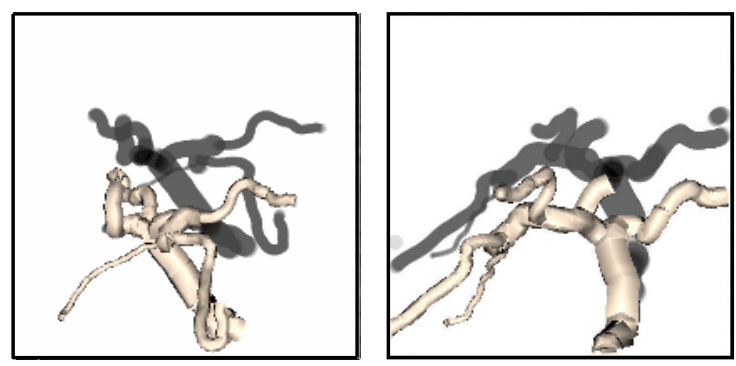

Fig. 2. An example of ideal-case simulated pseudoangiograms (gray), AP (left) and lateral (right). Also shown is a perspective projection of the 3D segmented vasculature (red).
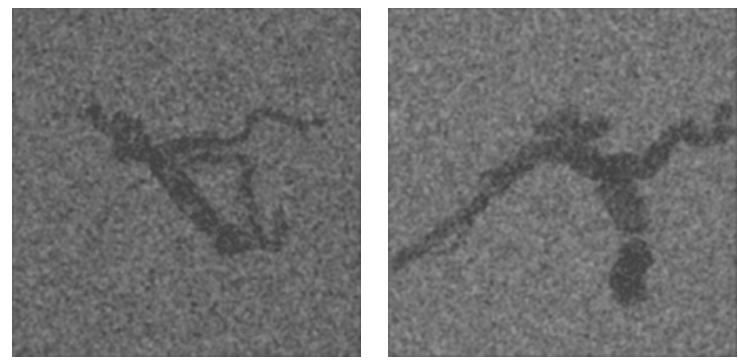

Fig. 3. Simulated angiograms with added noise. Vessels have the same pose as shown in Fig. 2 
generation. Mean 3D point placement errors were calculated for each case during each study. One result was reported per case when simultaneous registration was employed, and two results were reported per case (one each for Anterior-Posterior (AP) and Lateral images) when sequential registrations were employed.

\subsection{Clinical Studies}

A comparison of simultaneous and sequential two-view registration was also performed on three clinical cases using AP and lateral angiographic images obtained during TIPS procedures. Preoperative images of the 3D liver vasculature were acquired by CT (a Siemens Somatom Plus system was used with collimation $0.56 \times 0.56 \times 2.5 \mathrm{~mm}$ ) or MR (on a Siemens MagicVision 1.5T system with collimation $0.86 \times 0.86 \times 3 \mathrm{~mm}$ ). Voxel size was variable, but around $1.5 \times 1.5 \times 3 \mathrm{~mm}$.

Extraction of the portal venous tree from 3D image data involved 3 steps: definition of a seed point, automatic extraction of an image intensity ridge representing the vessel's central skeleton, and automatic determination of vessel radius at each skeleton point [7]. Vessels are represented as sets of 4-dimensional points with an $(\mathrm{x}, \mathrm{y}, \mathrm{z})$ spatial position and an associated radius.

A Siemens Neurostar biplane digital angiographic unit was used to obtain x-ray angiograms as biplane views, separated by approximately $90^{\circ}$. The fields of view ranged from $8.7^{\circ}$ to $16.5^{\circ}$. Images were captured and stored as 8 -bit $884 \times 884$ pixel images. Fig. 4 shows sample clinical images.

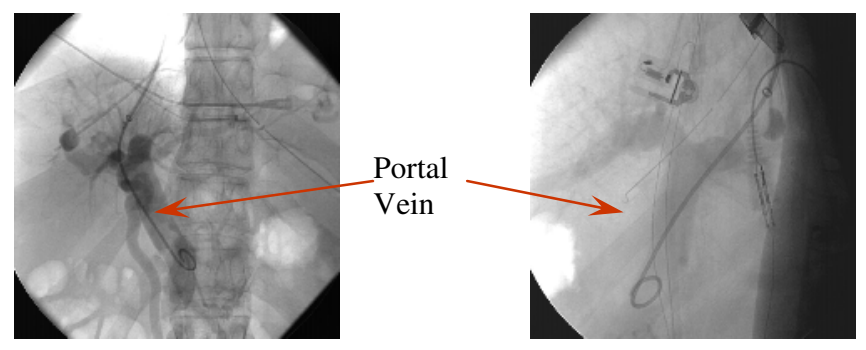

Fig. 4. AP (left) and lateral (right) abdominal angiograms obtained intra-operatively. Note the noisiness of the data and the significant projection overlap induced by wide-diameter vessels.

For patient studies, the relationship between the two fluoroscopic views was calibrated using a Plexiglas phantom containing a known arrangement of $5 \mathrm{~mm}$ diameter metallic spheres. The projection matrix for each view was calculated by taking an x-ray image of the phantom and minimizing the distance between observed pixel coordinates and ideal projection of each control point [8]. Intrinsic camera parameters were calculated using the same phantom.

\section{Validation of Registration Results}

The evaluation of clinical results is difficult since ground truth is unknown. Registration accuracy was estimated in these cases by reconstructing into 3D, a point that could be identified in both AP and lateral views, and comparing the location of 
this reconstructed 3D point to that of its corresponding point in the 3D model following registration. One subject ('Patient 1' in Table 2) had a metal clip in the liver as a result of previous surgery, and this clip was visible in the preoperative CT and on both projection views. The same subject also had 2 vessel branch-points that, with the help of an expert radiologist, could be associated on the AP and lateral projection views. Patients 2 and 3 had 3 and 2 vessel branch-points, respectively, that could similarly be associated on the AP and lateral views.

\section{Results}

\subsection{Phantom Studies}

For each test case, registration errors are presented for ideal pseudoangiograms, noisy pseudoangiograms and noisy pseudoangiograms without one-to-one vessel correspondences. For each of these pseudoangiogram pairs, accuracy is reported for simultaneous, sequential AP, and sequential lateral registrations.

Addition of noise to the image obscures small vessels and distorts vessel shape, making an accurate registration harder. The lack of one-to-one vessel correspondence is another source of error. For example, the image on the left in Fig. 5 shows a trimmed 3D model registered with the pseudoangiogram. On the right, the model has all branches present. The dark curves identify the position of previously deleted branches, thus highlighting the error.
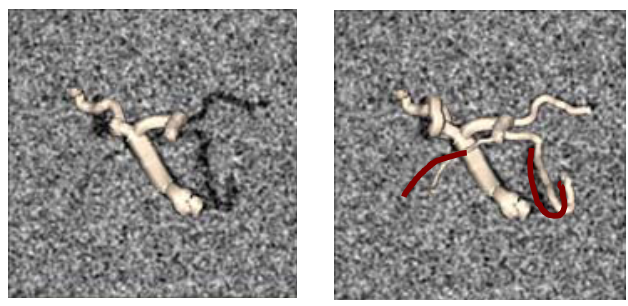

Fig. 5. Registration difficulties in presence of noise and without one-to-one correspondence

Vessel registration errors for the phantom studies, presented in Tables 1 and 2 and Fig. 6, 7 and 8, are calculated as described in section 2.1.

\subsection{Clinical Studies}

Table 3 contains the registration accuracy data for the clinical trials, calculated as detailed in section 2.4. For patient 1, the registration error given is the mean of the error over three points - a surgical clip and two vessel branch-points. For patients 2 and 3, the mean registration error for 3 and 2 branch-points, respectively, is presented. 
Table 1. Registration errors for phantom studies for the ideal and noisy pseudoangiograms. Errors are in $\mathrm{mm}$ and are presented in the 'mean (maximum)' format.

\begin{tabular}{|c|c|c|c|c|c|c|}
\hline \multirow{3}{*}{ Case } & \multicolumn{3}{|c|}{ Ideal pseudoangiograms } & \multicolumn{3}{|c|}{ Noisy pseudoangiograms } \\
\hline & \multicolumn{2}{|c|}{$\begin{array}{l}\text { Sequential } \\
\text { Registration }\end{array}$} & \multirow{2}{*}{$\begin{array}{l}\text { Simult- } \\
\text { aneous } \\
\text { Regn. }\end{array}$} & \multicolumn{2}{|c|}{$\begin{array}{c}\text { Sequential } \\
\text { Registration }\end{array}$} & \multirow{2}{*}{$\begin{array}{l}\text { Simult- } \\
\text { aneous } \\
\text { Regn. }\end{array}$} \\
\hline & $\mathrm{AP}$ & Lateral & & $\mathrm{AP}$ & Lateral & \\
\hline 1 & $1.1(2.1)$ & $1.1(2.1)$ & $0.3(0.6)$ & $4.3(4.8)$ & $0.7(1.7)$ & $0.5(1.9)$ \\
\hline 2 & $3.1(3.9)$ & $1.1(2.0)$ & $0.3(0.6)$ & $2.1(3.6)$ & $1.1(2.0)$ & $0.7(1.1)$ \\
\hline 3 & $1.0(2.2)$ & $0.3(0.5)$ & $0.3(0.5)$ & $1.4(2.0)$ & $5.0(5.5)$ & $0.9(1.2)$ \\
\hline 4 & $2.8(2.9)$ & $3.7(4.1)$ & $0.7(0.9)$ & $6.3(6.9)$ & $1.3(1.4)$ & $1.0(1.2)$ \\
\hline 5 & $3.1(5.3)$ & $1.1(1.5)$ & $0.2(0.2)$ & $1.7(2.2)$ & $1.7(2.2)$ & $1.1(1.6)$ \\
\hline 6 & $4.1(4.2)$ & $3.8(5.3)$ & $0.2(0.2)$ & $2.0(4.4)$ & $1.9(3.8)$ & $1.0(2.6)$ \\
\hline 7 & $0.6(1.0)$ & $0.6(1.0)$ & $0.2(0.2)$ & $8.2(9.2)$ & $3.4(4.5)$ & $1.1(1.4)$ \\
\hline 8 & $0.5(1.0)$ & $1.0(1.7)$ & $0.4(0.6)$ & $2.3(3.8)$ & $4.9(6.1)$ & $0.6(0.7)$ \\
\hline
\end{tabular}

Table 2. Phantom registration errors in $\mathrm{mm}$ for noisy pseudoangiograms without one-to-one correspondence

\begin{tabular}{|c|c|c|c|}
\hline \multirow{2}{*}{ Case } & \multicolumn{3}{|c|}{ Noisy pseudoangiograms without 1-to-1 correspondence } \\
\cline { 2 - 3 } & Sequential Registration & \multirow{2}{*}{$\begin{array}{c}\text { Simultaneous } \\
\text { Registration }\end{array}$} \\
\cline { 2 - 3 } & AP & Lateral & $0.5(1.1)$ \\
\hline 1 & $1.1(2.9)$ & $1.9(2.2)$ & $1.1(2.3)$ \\
\hline 2 & $3.4(5.5)$ & $1.1(2.0)$ & $1.0(1.4)$ \\
\hline 3 & $1.4(4.5)$ & $6.8(7.0)$ & $1.0(1.5)$ \\
\hline 4 & $5.3(7.4)$ & $5.0(6.9)$ & $1.2(2.0)$ \\
\hline 5 & $7.3(8.2)$ & $6.0(6.9)$ & $1.1(2.9)$ \\
\hline 6 & $3.8(5.4)$ & $7.7(8.7)$ & $0.8(1.4)$ \\
\hline 7 & $8.7(9.3)$ & $8.0(8.8)$ & $0.6(0.9)$ \\
\hline 8 & $1.2(1.9)$ & $4.2(6.8)$ & \\
\hline
\end{tabular}

Table 3. Registration errors in mm for clinical trials. Errors are in 'mean (maximum)' format.

\begin{tabular}{|c|c|c|}
\hline Patient & $\begin{array}{c}\text { Sequential } \\
\text { Registration }\end{array}$ & $\begin{array}{c}\text { Simultaneous } \\
\text { Registrations }\end{array}$ \\
\hline 1 & $6.1(7.2)$ & $1.9(2.12)$ \\
\hline 2 & $5.5(9.6)$ & $2.6(3.7)$ \\
\hline 3 & $4.0(4.8)$ & $2.4(2.5)$ \\
\hline
\end{tabular}



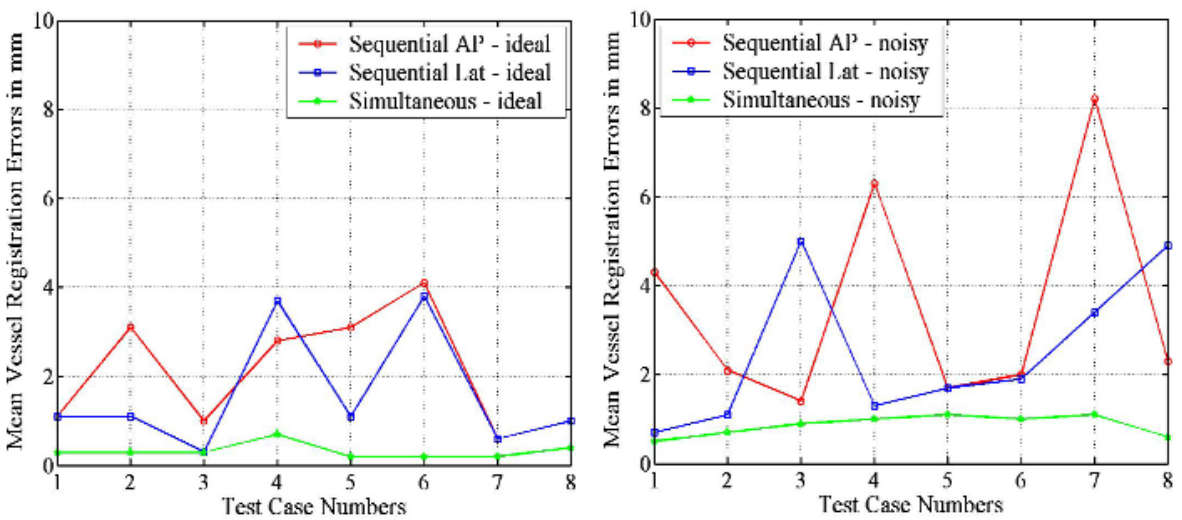

Fig. 6. Mean registration errors for the phantom studies with ideal angiograms

Fig. 7. Mean registration errors for the phantom studies with noisy angiograms

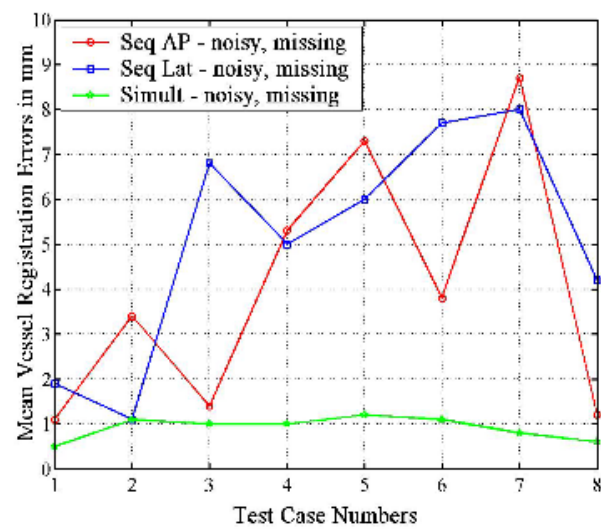

Fig. 8. Mean errors for phantom studies with noise and without one-to-one correspondence

\section{Discussion and Conclusion}

This paper compares the accuracy of simultaneous and sequential two-view registration in both phantom and clinical images of abnormal hepatic vasculature. The magnitude of the error we report for sequential-view registration is larger than the 1-2 $\mathrm{mm}$ error generally described in the literature [3,4,5]. Almost all prior studies evaluating 2D-3D vessel registration accuracy have used noiseless images, thin vessels, and one-to-one vessel correspondence, however. As shown by Figure 1, the images generated during TIPS are often of low quality and many vessels are thick the main branch of the portal vein may be over $1 \mathrm{~cm}$ wide. Thick vessels increase projection overlap and complicate both centerline and branch-point definition. The difficulty of accurate 2D-3D registration is thus high in this actual clinical situation. 
The current study details the behavior of the two registration methods with changes in image quality. Even under ideal conditions, one might expect simultaneous twoview registration to perform superiorly since with single-view registration, translation of an object along the depth axis produces relatively little change on projection. Simultaneous use of two orthogonal views allows each registration to correct the depth estimate of the other. Our phantom studies confirm this hypothesis, with improvement of accuracy by simultaneous registration even under ideal conditions.

The difference between the two approaches becomes more pronounced under conditions of noise and lack of one-to-one vessel correspondence - factors incorporated both in our phantom studies and in clinical data. Indeed, in two of the three clinical cases, the error in simultaneous registration was more than twice the magnitude of the error for sequential registration. This finding may result from the ability of one view to compensate for regional ambiguities in the other.

We conclude that simultaneous, two-view registration produces significantly more accurate results than sequential view registration. These findings should be applicable to a variety of $2 \mathrm{D}-3 \mathrm{D}$ registration metrics, and are of interest to those involved in the guidance of endovascular surgery.

\section{Acknowledgements}

This work was supported by R01 HL69808 NIH-HLB.

\section{References}

1. J.M. LaBerge, E.J. Ring, R.L. Gordon, J.R. Lake, M.M. Doherty, K.A. Somber, J.P. Roberts, N.L. Ascher, "Creation of Transjugular Intrahepatic Portosystemic Shunts with the Wallstent Endoprosthesis: Results in 100 Patients”, Radiology, 187(2):413-20, May 1993

2. J. Feldmar, G. Malandain, N. Ayache, S. Fernández-Vidal, E. Maurincomme and Y. Trousset, "Matching 3D MR Angiography Data and 2D X-ray Angiograms", CVRMedMRCAS 1997, Grenoble, France, pp.129-138

3. H.M. Chan, A.C.S. Chung, S.C.H. Yu, W.M. Wells, "2D-3D Vascular Registration between Digital Subtraction Angiographic (DSA) and Magnetic Resonance Angiographic (MRA) Images", IEEE International Symposium on Biomedical Imaging: From Nano to Macro, April 2004

4. Y. Kita, D. Wilson, J.A. Noble "Real-time Registration of 3D Cerebral Vessels to X-ray Angiograms" MICCAI 1997, pp. 1125-1133

5. A. Liu, E. Bullitt, S. Pizer "3D/2D Registration via Skeletal Near Projective Invariance in Tubular Objects", Medical Image Computing and Computer-Assisted Intervention, LNCS 1496: 952-963, MICCAI 1998

6. Y. Kita, D. Wilson, J.A. Noble, N. Kita "A quick 3D-2D Registration Method for a WideRange of Applications", Proceedings of the International Conference on Pattern Recognition, pp. 981-986, ICPR 2000

7. S. Aylward, E. Bullitt "Initialization, noise, singularities and scale in height ridge traversal for tubular object centerline extraction", IEEE-TMI 21:61-75, 2002

8. O. Faugeras “Three-Dimensional Computer Vision: A Geometric Viewpoint”, MIT Press 1996 